\title{
Achtung Lebensgefahr! Indirekte Effekte regionaler Arbeitslosigkeit auf Lebensweise und -qualität Gerd Grözinger*
}

Im Gefolge der Finanzkrise wird auch die unfreiwillige Erwerbslosigkeit in Deutschland wieder - vermutlich sogar erheblich - ansteigen. Es ist deshalb von erneuter Aktualität und Dringlichkeit, einen genaueren kritischen Blick auf die Gesamtwirkungen der Arbeitslosigkeit zu werfen. Im Folgenden wird dies für die Dimension regionaler Einflüsse geschehen und zwar in Bezug auf die Wirkung auf die Lebensweise und -qualität der Menschen. Damit soll nicht ausgesagt werden, dass andere mögliche Fokussierungen nicht gleichfalls ihre Berechtigung haben. Denkbar wäre, beispielsweise, Familien, Netzwerke oder materielle Flüsse, etwa bezüglich Mindereinnahmen an Steuern oder Sozialabgaben, in den Mittelpunkt zu stellen. Aber der hier benutzte Fokus scheint besonders erkenntnisträchtig, wie gezeigt werden soll. Denn es gibt dazu bereits etliche Untersuchungen, die jedoch oft wenig bekannt und auch nie zusammengetragen worden sind. Eine solche - vermutlich auch noch lange nicht vollständige ${ }^{\mathrm{I}}$ - Gesamtschau lässt die Dimension des Problems aber erst deutlich hervor treten.

Im ersten Abschnitt wird daher angesprochen, welche Regionalebenen in Deutschland für eine solche Fragestellung möglich und besonders geeignet sind. Anschließend folgt ein Überblick über verschiedene relevante Wirkungszusammenhänge. Danach wird gefragt, ob die jeweilige Arbeitslosigkeit die Lebenserwartung in der Region beeinflusst. Dies hat in der bisherigen Debatte noch keine Rolle gespielt, ist aber durchaus von einiger Bedeutung, wie aufgezeigt wird. Abschließend soll versucht werden, die Befunde in einen größeren Zusammenhang aktueller sozialwissenschaftlicher Diskussionen zum Thema Ungleichheit und Sterblichkeit zu stellen.

* Universität Flensburg. Für kritische Hinweise danke ich den TeilnehmerInnen des Interdisziplinären Kolloquiums an der Universität Flensburg, der Tagung Health Inequalities III an der Universität Bielefeld, der Sommerakademie des Studienwerks der Heinrich Böll Stiftung sowie der Seminare am Inter-University Center Dubrovnik und am Institute for European Studies an der University of British Columbia, Vancouver und nicht zuletzt auch der Redaktion der Intervention für Verbesserungsvorschläge.

I Für entsprechende Hinweise auf empirische Studien zu weiteren Feldern, z.B. zur Kriminalität oder zur Fertilität, wäre ich dankbar. Bisher sind mir hier nur erste bescheidene Ansätze aufgefallen, die allerdings in die gleiche Richtung gehen und einen negativen Wirkungseffekt aufweisen (z.B. zum letzteren Ragnitz 2006).

(C) INTERVENTION 6 (I), 2009, I2-24 


\section{Länder, Regionen, Kreise/Gemeinden als Untersuchungsebene?}

Von Regionalinformationen auf individuelles Verhalten zu schließen, wie es etwa Durkheim noch recht selbstverständlich unternahm, hatte lange in den Sozialwissenschaften keinen guten Ruf mehr. Kritiker witterten sofort einen `ökologischen Fehlschluss` (Robinson I950), weil sich doch herausgestellt hatte, dass die statistisch gemessene Eigenschaft eines Gebiets nicht notwendiger Weise sich auf menschliches Verhalten übertragen ließ. Wenn also etwa Schweizer Kantone mit überwiegend protestantischer Bevölkerung eine relativ niedrigere Kinderzahl hatten, so ist es noch lange nicht automatisch sicher, dass darin lebende Protestanten auch weniger Kinder als die dortigen Katholiken haben. Diese zeitweise völlige Abkehr von regional argumentierenden Arbeiten hat sich durch das Aufkommen erfolgreicher `Nachbarschaftsstudien $`$ allerdings wieder geändert. In diesen Studien konnte - oft auch mit durchaus avanciertem methodischem Instrumentarium - die Existenz von soliden Umfeldeinflüssen dokumentiert werden. Besonders fruchtbar war dabei die Kombination von Individual- mit Umfelddaten. Eine Auszählung hierzu ergab:

»In den Jahren 1992 bis 1996 wurden durchschnittlich I,6 Artikel pro Jahr mit den Schlagwörtern ımultilevel und sneighborhood in den Fachzeitschriften veröffentlicht, die im Social Sciences Citation Index erfasst sind. In der Periode von 1997 bis 200I waren es schon I2,2 Artikel jährlich und in den Jahren 2002 bis 2006 im Mittel 53,0 Artikel« (Nonnenmacher 2007: 493).

Und auch Arbeiten mit reinem Regionalbezug werden jetzt wieder interessant, weil dadurch zumindest Hinweise auf sonst unentdeckt gebliebene Wirkmechanismen zu gewinnen sind.

Offen bleibt aber weiterhin, welche räumliche Kontextebene zum Nachweis welchen Effektes angemessen ist. Die Skala reicht hier von einem Plädoyer für möglichst kleinräumige Untersuchungen in Einstellungsfragen, wie etwa der Kriminalitätsfurcht (Nonnenmacher 2007), bis zum von einigen AutorInnen bevorzugten Vergleich ganzer Nationen bezüglich Morbidität und Mortalität (Wilkinson/Pickett 2007).

Für die ergänzende eigene Untersuchung war eine Entscheidung über die angemessene Analyseebene zu treffen. Diese Liste kann auch zur besseren Einschätzung der anderen vorgestellten Studien dienen. Folgende Möglichkeiten bestehen, wenn es um deutschlandweite (und nicht etwa einzelne gemeindebezogene) Daten geht:

- $\quad$ 16 Länder mit einer Spannbreite an Bevölkerung von 0,7 - I8 Mill. Einwohner;

- $\quad 97$ Raumordnungsregionen mit Einheiten zwischen 0,3- 3 Mill. Einwohner;

- $\quad 439$ Kreise und Städte, deren Bevölkerungsstärke zwischen o,o5 und 3 Mill. Einwohner liegt.

Es wurde die mittlere Ebene der Raumordnungsregionen gewählt. Hier besteht erstens der Vorteil, dass Arbeitsmarktdaten weniger durch Berufspendler gestört sind. Zweitens sind die meisten der vorgestellten anderen Analysen auf dieser oder einer zumindest sehr ähnlichen Ebene verfasst, so dass eine direkte Korrespondenz der Ergebnisse gegeben ist. Und drittens 
wurde die Berechnung auf die Beeinflussung der Lebenserwartung nach einer Arbeit über Italien modelliert, der eine in etwa vergleichbare regionale Aufteilung zu Grunde lag.

Wenn andere Arbeiten mit der Ebene von Kreisen/Gemeinden arbeiten, wird dies jeweils benannt werden. Analysen auf der Ebene der Bundesländer werden dagegen nur ausnahmsweise berücksichtigt, da die geringe Zahl der Fälle es schwer macht, diesbezüglich abgesicherte statistische Aussagen zu treffen.

\section{Multiple Wirkungen regionaler Arbeitslosigkeit}

Wer arbeitslos wird, verliert vor allem Einkommen. Aber was ist mit denen, die noch Arbeit haben? Ist deren Einkommenssituation davon unbeeinflusst, ob in ihrer Region eine hohe oder eine niedrige Arbeitslosigkeit herrscht? In Anlehnung an eine paradigmatische erste Untersuchung über die USA (Blanchflower/Oswald 1994) mit vielen folgenden Bestätigungen in anderen Ländern wurde auch in Deutschland nach einem solchen regionalen Einfluss gesucht, und man wurde fündig. Erste Wirkung neben dem Einkommensverlust ist die Beobachtung, dass das regionale Lohnniveau negativ von der Höhe der regionalen Arbeitslosigkeit abhängt. Auf Grundlage der Beschäftigtenstatistik des IAB konnte dies sowohl für Ostdeutschland (Baltagi et al. 200o) wie mehrfach für Westdeutschland (zuletzt: Baltagi et al. 2008) gezeigt werden. Wohl wegen der zentralisierten Art der Lohnfindung ist dabei der Einfluss im Westen geringer. In beiden Fällen bildeten Kreise die Analyseebene.

Auch der zweite indirekte Effekt beruht auf Daten des IAB, diesmal aber auf dem Betriebspanel, das regionalisierbar ist. Hier wird ein negativer Einfluss der Arbeitslosigkeit auf Weiterbildungsaktivitäten von Betrieben beobachtet (Martin/Düll 200o). Unternehmen haben es nicht nötig, besonders in die Humankapitalausstattung ihrer Mitarbeiter zu investieren, wenn es genug freie Alternativen auf dem Arbeitsmarkt gibt. Bei ungelernten Arbeitskräften dreht sich dabei das Bild um, vermutlich den dann einsetzenden öffentlichen Fördermaßnahmen geschuldet.

Wenn sowohl Löhne wie betriebliche Bildungsmaßnahmen in Gebieten mit höherer Arbeitslosigkeit relativ niedriger als anderswo sind, dann sollten Arbeitskräfte eine gewisse Motivation haben, in erfolgreichere Gegenden umzuziehen um sich dort wirtschaftlich zu verbessern. Stimmt diese Annahme neoklassischer ökonomischer Wanderungstheorie, die von einem stark rationalen Verhalten ausgeht? Die Antwort ist Nein; man beobachtet in Deutschland das Gegenteil. Der dritte Effekt regionaler Arbeitslosigkeit ist die Abnahme der Mobilitätsbereitschaft der Bevölkerung bei höherer regionaler Arbeitslosigkeit (Windzio 2004). Auch hier wurde wieder die Kreisebene als Analyseeinheit gewählt, und die Regionalstichprobe des IAB bildete erneut die Datenbasis. Dieser Individualdatensatz erlaubt einige zentrale Merkmale von Personen mit Kontextdaten zu verknüpfen und führte zu dem Ergebnis, dass durchaus gebietsmäßig zu verortende `Arbeitslosenfallen existieren, aus denen es vielen schwer fällt zu entkommen. Zu vermuten ist eine `depressive Attribution‘, wie sie in der Sozialpsychologie beschrieben wird (Hewston/Antaki I990).

Mit einem solchen Erklärungsansatz ist man bereits bei den eher subkutanen Wirkungen. Hier sind eine ganze Reihe von stark wirkenden Kontexteinflüssen gefunden worden. So 
wurde auf der Basis des Sozio-ökonomischen Panels (SOEP) untersucht, inwiefern die Arbeitslosenquoten der Raumordnungsregionen eine Wirkung auf zentrale Zufriedenhe itsdimensionen haben. Das SOEP ist eine große repräsentative Wiederholungsbefragung privater Haushalte in Deutschland, die die Grundlage zahlreicher sozialwissenschaftlicher Untersuchungen bildet, und das sich auch hier als ergebnisträchtig erwiesen hat. Vierter Effekt ist eine erhebliche negative Beeinflussung allgemeiner Lebenszufriedenheit als auch der Gesundheitszufriedenheit durch eine höhere regionale Arbeitslosigkeit, die bestehen bleibt, wenn für individuelle Merkmale kontrolliert wird (Grözinger/Matiaske 2004). Die allgemeine Lebenszufriedenheit wird dabei zunehmend als zentraler Maßstab einer politischen Zielfunktion akzeptiert (z.B. Layard 2005), so dass eine hier vorhandene negative Wirkung für eine politische Einschätzung besonders schwer wiegt. Und die subjektive Gesundheitszufriedenheit hat sich auch im SOEP als guter Indikator für den wirklichen Gesundheitszustand heraus gestellt (Schwarze et al. 2002), lässt also höhere Werte an Morbidität und Mortalität erwarten. Zwar nur auf der Ebene der Bundesländer vorhanden, aber dafür über eine längere Zeitreihe hinweg, hat der Nachweis der negativen Beeinflussung der Lebenszufriedenheit durch die Gebietsarbeitslosigkeit mit Hilfe des SOEP noch einmal Bestätigung durch eine neuere Studie erfahren (Luechinger et al. 2008).

Bei einer Bedrohung von Status und Einkommen sind neben der Zunahme depressiver und psychosomatischer Verhaltensweisen auch nach außen gerichtete aggressive Verhaltensweisen, vor allem gegenüber zu Sündenböcken gemachten Gruppen nicht unwahrscheinlich. Aber es gibt für Deutschland erstaunlich wenig Arbeiten dazu, die diese Annahme auch einer statistischen Analyse unterzogen haben. Eine Ausnahme ist ein Test auf der Grundlage von Daten des Bundeskriminalamts bezüglich der - wenn man sich nicht nur auf Gewalttaten beschränkt, erstaunlich hohen - Anzahl von Straftaten mit rechtsradikalem Hintergrund. Diese konnten zwar nur auf der Bundesländerebene ausgewertet werden, erlaubten aber die Integration zahlreicher regionaler Kontrollvariablen. Die fünfte zu berichtende Zusatzproblematik ist somit ein stark positiver Zusammenhang von Arbeitslosigkeit und Rechtsradikalismus (Falk/Zweimüller 2005). Die Autoren fanden dabei auch noch heraus, dass ein allgemeiner Ost-West-Unterschied nur noch in geringem Maße nachweisbar ist, wenn die Arbeitslosigkeitsdifferenz in das Modell eingeführt wird: ganz überwiegend erklärt diese dann den Unterschied im politischen Verhalten.

Besonders bedrückend sind auch eventuelle Langzeitwirkungen regionaler Defizite bei den nächsten Generationen. Den ersten systematischen Nachweis einer solchen Beziehung verdanken wir überraschenderweise einer Einrichtung die bisher in der sozialwissenschaftlichen Forschung eher klandestin gewirkt hat: der Bundeswehr. Aber durch die flächendeckend durchgeführten psychologischen Tests bei jungen männlichen Wehrpflichtigen liegt ein einzigartiger Datensatz vor, dessen Auswertung auf Regionalausflüsse Überraschendes zu Tage förderte. Eine sechste Wirkung negativer Art besteht nämlich im Rückgang der gemessenen Intelligenz in Regionen mit höherer Arbeitslosigkeit (Ebenrett et al. 2003). Als Untersuchungseinheit dienten die 83 Kreiswehrersatzämter, also eine Aufteilung, die der nach Raumordnungsregionen recht ähnlich ist. In einem Pfadmodell wurde die Arbeitslosigkeit dabei als bedeutendste Variable für die IQ-Differenzen isoliert, gefolgt von der Abwanderung 
Höherqualifizierter, einem nicht als unabhängig zu bewertenden, sondern zusätzlichen mittelbaren Effekt regional schlechter Arbeitsmärkte.

Die Autoren der Studie merkten auch an, dass die ausschließliche Zentrierung der deutschen PISA-Debatte auf bildungspolitische Fragen bei solchen Ergebnissen zu relativieren wäre und ob eine Bekämpfung der Arbeitslosigkeit nicht einen ähnlichen hohen Rang zur Sicherung zukünftigen Humankapitals einnehmen sollte. Das hat ihnen im spädagogisch-administrativen Komplex Deutschlands keine Freunde gemacht. Vermutlich wurden Sie deshalb zum künftigen Schweigen verpflichtet. ${ }^{2}$ Aber sie dürfen sich zugute halten, dass als Reaktion auf diese unwillkommene Herausforderung auch die umfangreiche PISA E-Stichprobe von 2003 einer detaillierten Regionalanalyse auf Kreisebene unterzogen wurde, wieder mit interessantem Ergebnis hinsichtlich des Zusammenhangs von Arbeitslosigkeit und Schulleistung (Baumert et al. 2005). Siebenter Effekt ist nämlich die negative Wirkung regionaler Arbeitslosigkeit auf Schulleistungstests. Für Mathematik, dem Schwerpunkt dieser Befragungswelle, wurden maximale Leistungsdifferenzen aufgrund der Arbeitsmarktsituation im Umfang von etwa 30 Punkten errechnet, das entsprich etwa dem Lernfortschritt eines Jahres. Dazu kam ein zusätzlicher negativer Einfluss durch den regional unterschiedlichen Sozialhilfebezug, der wiederum zur Arbeitslosigkeit in einer positiven Beziehung steht. ${ }^{3}$

\section{Ein weiterer Effekt: Lebenserwartung}

Bei der Fülle der Nachweise eines starken Einflusses der Gebietsarbeitslosigkeit auf viele Dimensionen menschlichen Handelns, sollte da nicht auch die Gesundheit - und als Langfristfolge: die Sterblichkeit - betroffen sein? Erstaunlicher Weise ist dies bisher wenig untersucht worden. Mit Ausnahme der Analyse der Ost-West-Differenzen in Folge des Einigungsprozesses waren regionale Unterschiede kaum ein Thema in Deutschland.

Dabei ist das Bild der Abhängigkeit der durchschnittlichen Lebenserwartung von der regionalen Arbeitslosigkeit durchaus beeindruckend. Beispielhaft deutlich wird dies in den beiden folgenden Grafiken, die den Zusammenhang für Männer und Frauen getrennt darstellen und jeweils noch eine Regressionsgerade mit aufführen. Die Werte wurden der INKAR-CD des Bundesamts für Bauwesen und Raumordnung entnommen (Bundesamt für Bauwesen und Raumordnung 2004). Die Buchstaben stehen für Westdeutschland (W), Ostdeutschland (E) sowie die einzig gemischte Region Berlin (B).

2 Ein Indiz dafür ist, dass meine mehrfachen Versuche mit E-Mails - das Sozialwissenschaftliche Institut der Bundeswehr weist ganz fortschrittlich eine Homepage mit Kontaktformular auf - sowie Briefen Aufklärung zu eventuell geplanten weiteren Studien zu dem Thema oder Zugangsmöglichkeiten zu den Daten zu erlangen, kein einziges Mal zu irgendeiner Reaktion führten.

3 Vermutlich ist der Effekt noch unterschätzt, da in dem Modell zugleich noch eine West-OstWirkung eingeführt wurde, die eine erhebliche weitere Wirkung hat. Aber der Umfang dieses Einflusses lässt sich schwer einschätzen und leider schon gar nicht überprüfen: Das PISA-Konsortium stemmt sich beharrlich dagegen, dass die regionalisierten Daten der Wissenschaft für weitere Analysen zur Verfügung gestellt werden. 
Abbildung I: Lebenserwartung und regionale Arbeitslosenquote in \% - Männer

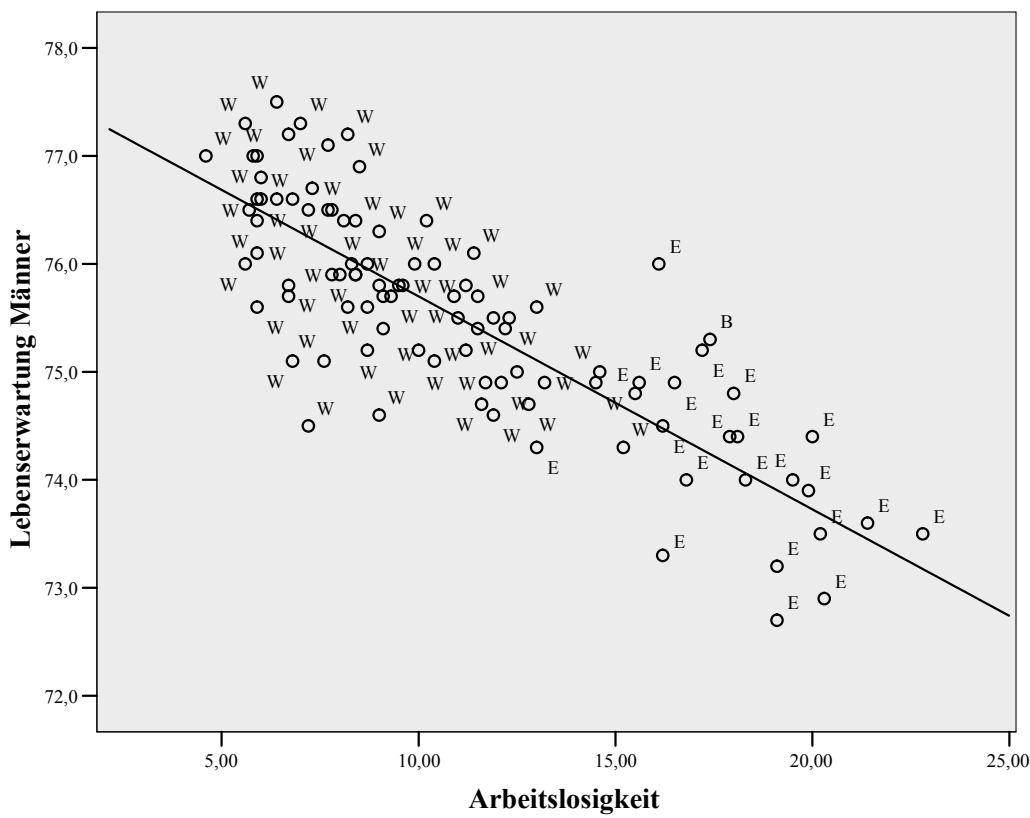

Abbildung 2: Lebenserwartung und regionale Arbeitslosenquote in \% - Frauen

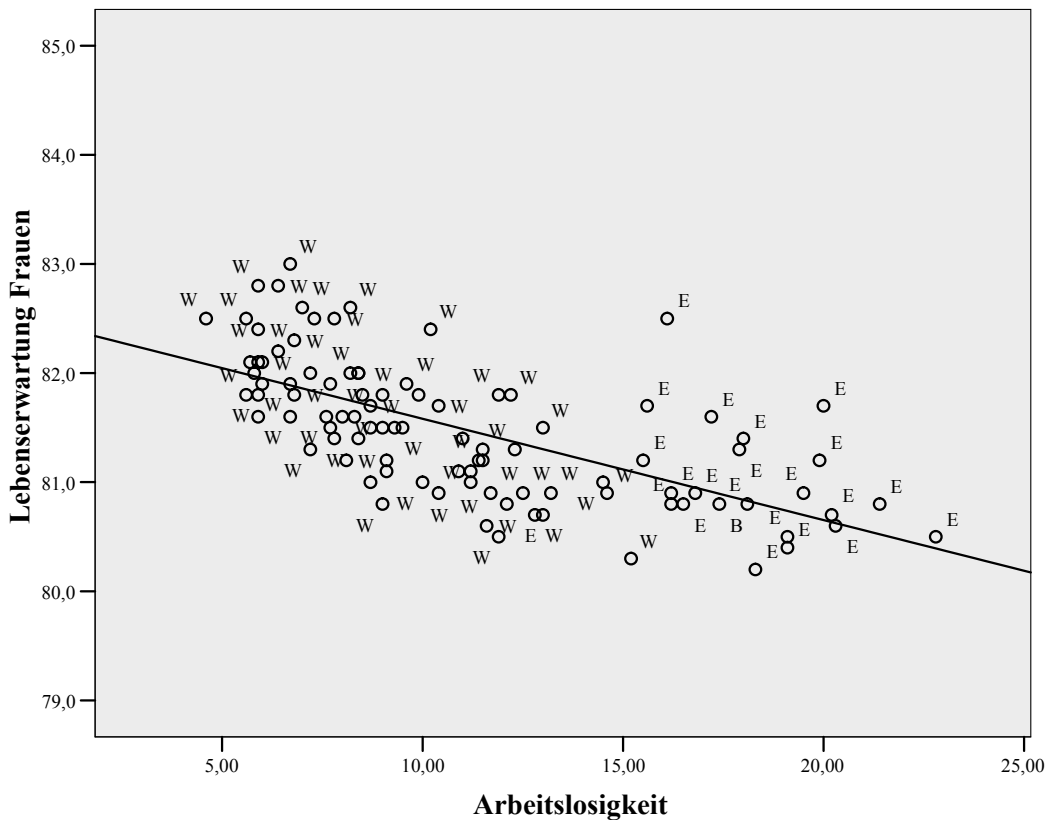


Es fallen sofort drei Zusammenhänge auf: (a) Es gibt einen starken und eindeutigen Zusammenhang von Lebenserwartung und Arbeitslosenquote; (b) Ost und West überlappen sich kaum; (c) auch getrennte Darstellungen für Ost und West würden jeweils für sich einen solchen Zusammenhang zeigen. Diese drei Aussagen treffen für beide Geschlechter gleichermaßen zu, aber die Steigungsgrade der Regressionsgeraden differieren. Während sich für Frauen die Lebenserwartung zwischen den Regionen mit der niedrigsten und der höchsten Arbeitslosenquote um 2,3 Jahren unterscheiden, sind es bei Männern 4,8 Jahre. Solche geschlechtsspezifischen Differenzen in Bezug auf soziale Einflüsse werden häufig beobachtet, wobei zumeist auf eine niedrigere Integration von Frauen im Arbeitsmarkt verwiesen wird (Robert-Koch-Institut 2005).

Die Daten beziehen sich auf das Jahr 2002. Dass die Arbeitslosigkeit nur eines Jahres als wirkend unterstellt werden darf, liegt an der großen Strukturkonstanz im regionalen Bild. Zwar mag über eine etwas längere Periode, wie etwa die Phase von 1995-2004, die Gesamtarbeitslosigkeit durchaus schwanken und damit verändern sich natürlich auch die einzelnen regionalen Kennziffern, aber die relative Positionierung untereinander bleibt gut erhalten (Legewie 2008, Fn. Io).

Ein möglicher Einwand, dass das oben gezeigte Bild nur eine Scheinbeziehung darstellen könnte, betrifft den unterschiedlichen Altersaufbau, wie er z.B. durch Wanderungen zustande kommt. Das Bedenken wäre korrekt, wenn hier die Gebietssterblichkeit angegeben wäre. Als Grundlage der hier durchgeführten Berechnungen dient aber die statistische Lebenserwartung, ein Indikator, der den Altersaufbau in der Konstruktion schon mitberücksichtigt und seinen verzerrenden Einfluss damit neutralisiert. Es gilt weitere kritische Überlegungen zu berücksichtigen. Die Argumentation mittels einfacher Korrelationen läuft immer Gefahr, andere eventuell zentrale Variablen zu vernachlässigen. Zwei zusätzliche Dimensionen müssen auf jeden Fall für einen vollständigeren Test mitberücksichtigt werden: das durchschnittliche Wohlfahrtsniveau und der Bildungsstand. Eine solche Erweiterung hat sich als Standard durchgesetzt, und wird z.B. in einer italienischen Arbeit benutzt, die hier als methodisches Vorbild dient (De Vogli et al. 2005). Das Wohlfahrtsniveau wird in der folgenden Berechung als BIP/Kopf bestimmt und ist ebenfalls der INKAR-CD entnommen. Für das regionale Humankapital gibt es dagegen keine amtlichen Statistiken. Hier konnte aber auf die Ergebnisse einer vom Autor initiierten Arbeit zurück gegriffen werden, in der u.a. für 200I die regionalisierte IAB-Beschäftigtenstichprobe auf Humankapital ausgewertet wurde (Tarazona 2007). ${ }^{4}$

Im Folgenden werden die Ergebnisse der entsprechenden Regressionsberechnungen dargestellt. Sie sind, wegen der unterschiedlichen Lebenserwartungen wieder nach Männern und Frauen getrennt aufgeführt. Zusätzlich ist gesondert jeweils noch die Situation nur für Westdeutschland allein dargestellt. Damit soll gezeigt werden, dass das Resultat nicht auf einem Ost-West-Effekt beruht, sondern auch in der sozialstrukturell viel stabileren alten Bundesrepublik nachweisbar bleibt.

4 Für die damalige finanzielle Unterstützung in Gestalt eines Forschungsstipendiums ist dem Rat für Sozial- und Wirtschaftsdaten zu danken. 
Tabelle I: Lebenserwartung (OLS-Regression)

\begin{tabular}{l|c|c|c|c}
\hline \multirow{2}{*}{} & \multicolumn{4}{|c}{ Standardisierte ß-Koeffizienten } \\
& Deutschland & Westdeutschland & Deutschland & Westdeutschland \\
\hline Arbeitslosigkeit & $-0,966^{* * *}$ & $-0,738^{* * *}$ & $-0,842^{* * *}$ & $-0,707^{* * *}$ \\
\hline BIP/Kopf & $-0,088$ & $-0,079$ & $-0,035$ & 0,038 \\
Bildung & $0,370^{* * *}$ & $0,452^{* * *}$ & $0,254^{* *}$ & 0,137 \\
Konstante & 62,841 & 65,037 & 77,096 & 81,794 \\
N & 97 & 75 & 97 & 75 \\
\hline $\mathbf{R}^{2}$, adj. & 0,781 & 0,610 & 0,461 & 0,525 \\
\hline
\end{tabular}

Anmerkung: Signifikanzniveau: < 0, OOI $\left(^{(* *}\right),<0,05\left(^{* *}\right)$

Das Ergebnis ist sehr einheitlich. Auf die Lebenserwartung von Männern wie Frauen wirken vor allem die regionale Arbeitslosigkeit und dann noch das Bildungsniveau. Wie auch schon in Italien beobachtet, löst sich der scheinbar starke Zusammenhang von Wohlfahrtsniveau und Lebenserwartung auf, wenn Bildung in das Modell mit aufgenommen wird. Der Erklärungsanteil ist recht gut, wie das $\mathrm{R}^{2}$ zeigt, es werden bei Frauen etwa die Hälfte, bei Männern ca. zwei Drittel der Varianz durch diese wenigen Variablen erklärt.

Die Wirkung der Gebietsarbeitslosigkeit ist in allen vier Fällen hochsignifikant, die der Bildung nur zwei Mal und ein Mal noch auf der 5\%-Ebene signifikant. Auch die Stärke des Einflusses unterscheidet sich. Durch die standardisierten Betawerte lassen sich die Werte für die einzelnen Variablen direkt miteinander vergleichen. Und hier wirkt bei Gesamtdeutschland die Arbeitslosigkeit bei beiden Geschlechtern etwa drei Mal so stark wie die Bildung und in Westdeutschland bei Männern etwa doppelt, bei Frauen (aber mit dann fehlender Signifikanz im Bildungsniveau) etwa fünf Mal so stark.

Nun ist die statistische Lebenserwartung zwar eine Kollektivgröße par excellence und kann deshalb auch nicht auf eine Individualebene herunter gebrochen werden. Aber das entbindet einen nicht davon, nach weiteren Indizien zu forschen, ob der hier aufgezeigte Zusammenhang auch mit anderen Daten oder Ansätzen bestätigt werden könnte, wobei besonders natürlich eine Absicherung durch Individualdaten wertvoll wäre.

Neben der vorne erwähnten Arbeit zur Gesundheitszufriedenheit (Grözinger/Matiaske 2004), bei der wenigstens eine gewisse Beziehung zur Lebenserwartung unterstellt werden darf, gibt es $\mathrm{m}$. W. nur noch eine neuere Arbeit, die sich dem Thema einer Beeinflussung von Mortalität durch die regionale Arbeitslosenquote direkt gewidmet hat. Dort wurden Sterbedaten der Deutschen Rentenversicherung für deutsche Männer ab 65 Jahren ausgezählt, was einige individuelle Merkmale wie Alter und Rentenhöhe zu berücksichtigen erlaubte. Allerdings konnten die Kontextinformationen nur auf der Ebene der Bundesländer integriert werden, auch ist die Arbeitslosigkeit nicht im Niveau sondern ausschließlich in der Entwicklung berücksichtigt und noch dazu wurde sie nur binär kodiert. Trotzdem ergab sich 
ein eindeutig steigernder Einfluss der Gebietsarbeitslosigkeit auf die Sterbewahrscheinlichkeit (Kibele 2008).

\section{Theoretische Einordnung}

Seit etwas mehr als einer Dekade gibt es eine lebendige Debatte um die Wirkung von Ungleichheit auf Gesundheit und Sterblichkeit. Vor allem angestoßen durch die Monografie eines britischen Sozialmediziners (Wilkinson 1996) sind sehr bald zahlreiche Arbeiten (z.B. Kawachi et al. 1999, Wilkinson 2005) entstanden, die sich mit folgender These auseinander setzten:

»Unter den modernen Industriegesellschaften sind nicht die reichsten Gesellschaften die gesündesten, sondern diejenigen mit den geringsten Einkommensunterschieden zwischen Arm und Reich. Soziale Ungleichheit und relative Armut sind außerordentlich wirksam: Sie steigern die Todesraten« (Wilkinson 1996: I).

Verständlich, dass eine solch provokante Aussage viele Reaktionen, vor allem auch in der Form empirischer Studien nach sich gezogen hat. Etwa zehn Jahre später zählt eine Überblicksarbeit schon I68 Arbeiten; 70\% von ihnen bestätigen die These der schädlichen Wirkung von Ungleichheit (Wilkinson/Pickett 2006). Und bei den Ausnahmen lässt sich oft angeben, warum hier die Wahrscheinlichkeit eines statistischen Zusammenhangs sinkt, nämlich dann wenn z.B. ein enger Zeitraum betrachtet wird, in dem die Ungleichheit zunimmt, aber zugleich die Altersarmut zurückgeht oder wenn dort neue effektivere Behandlungsmethoden bei der wichtigen Gruppe der Herz- und Kreislaufkrankheiten eingeführt wurden.

In Deutschland hat diese internationale Diskussion bisher kaum einen Widerhall gefunden. Auch regional orientierte Studien zur Gesundheit beschäftigen sich hier - mit wenigen Ausnahmen (Cromm/Scholz 2002) - ganz überwiegend mit der Variation individueller Risiken wie Rauchen, Ernährung etc. Selbst in der aktuellsten Zusammenstellung des Stands der Forschung in der Gesundheitssoziologie wird die Ungleichheitsthese von Wilkinson et al. kaum erwähnt, geschweige denn empirisch getestet (Wendt/Wolf 2006). Wer aber aus lauter Furcht vor einem `ökologischen Fehlschluss` die Kontextebene vernachlässigt, läuft dafür Gefahr, den symmetrischen Fehler eines ratomistischen Fehlschlusses` (Diez-Roux 2002) zu begehen, indem Umwelteinflüsse rein individuellem Verhalten zugeschrieben werden.

Nun bezieht sich diese zumindest anderswo geführte Debatte auf Ungleichheit, doch wie ist der Bezug zur Arbeitslosigkeit? Zwar kann hier sicher von starken Wechselwirkungen ausgegangen werden, aber sind sie so eng, dass die beiden Dimensionen austauschbar sind? Leider ist die Wirkung regionaler Arbeitslosigkeit viel weniger untersucht worden als die von Ungleichheit. Auch die vorne erwähnte Arbeit über Italien widmet sich der Ungleichheitsdimension, nicht jedoch der Arbeitslosigkeit (De Vogli et al. 2005).

Es gibt jedoch eine interessante Arbeit, die beides im Vergleich getestet hat, und das gleich noch für zwei verschiedene Länder, die USA und Kanada (Sanmartin et al. 2003). Das Ergebnis war, dass in den USA die Einkommensunterschiede besser die Regionaldifferenz 
in der Sterblichkeit erklärten, in Kanada jedoch die Arbeitslosigkeit. Die Autoren erklärten dies mit dem jeweils dominanten Muster an Exklusion: hier ein Arbeitsmarkt mit niedriger Zugangsbarriere aber eventuell schlechtem Einkommen und magerer sozialer Sicherung, dort ein besserer Schutz für Arbeitskräfte, aber dafür ein restriktiverer Arbeitsmarkt mit sich verfestigender Erwerbslosigkeit. Da Deutschland in Bezug auf Sozialstaatlichkeit sicher eher Kanada und nicht den USA ähnelt, könnte es also durchaus sein, dass auch hier die Arbeitslosigkeit den größeren Beitrag zur Erklärung regionaler Differenzen in der Lebenserwartung leistet. Aber das wird sich erst in Zukunft testen lassen, wenn die heute noch fehlenden regionalen Ungleichheitsmaße einmal vorliegen.

Wie sich Ungleichheit konkret in schlechtere Gesundheit und dann in höhere Sterblichkeit umsetzt, ist natürlich auch immer Gegenstand dieser Debatte gewesen. Sie soll hier aus Platzgründen nicht nachgezeichnet werden (siehe dazu Grözinger 2002). Aber ein vielleicht besonders wirkungsreicher Transfermechanismus verdient hervorgehoben zu werden, nämlich die negative Wirkung auf das Sozialkapital. Dies impliziert einen Rückgang gesellschaftlicher Aktivitäten, was eine erhebliche Bedeutung für die wahrgenommene Qualität der Lebensbedingungen hat (Kawachi et al. 1998). Dies würde auch zu einem Ergebnis passen, das Prognos gerade bezüglich der erstmalig erhobenen Regionalverteilung bürgerschaftlichen Engagements publiziert hat: die stärkste aller (positiven wie negativen) Korrelationen besteht mit der Arbeitslosigkeit, hier erwartungsgemäß negativ (Prognos 2008).

Schließlich sollte noch betont werden, dass die (berechtigte) Furcht, in Gebieten höherer Arbeitslosigkeit als Erwerbstätiger in Zukunft selbst vom Verlust der Arbeit bedroht zu sein, seine nachweislichen Wirkungen hat. In Deutschland ist die Lebenszufriedenheit der Beschäftigten im Privatsektor erheblich stärker als die der Beschäftigten im öffentlichen Dienst von der regionalen Arbeitslosenquote und ihrer Entwicklung betroffen. Und die strukturell anfälligere Gruppe sorgt sich mehr um die Arbeitsplatzsicherheit und die eigene ökonomische Situation wenn die Arbeitslosigkeit zunimmt (Luechinger et al. 2008). Aber auch der öffentliche Dienst bleibt nicht unberührt: Auch die hier Arbeitenden haben Familie, Nachbarn, Freunde, die von einer schwierigen Arbeitsmarktsituation betroffen sind oder es bald sein könnten, man spürt und sieht die vielen Zeichen der ökonomischen Krise in der Region. Dass solche `Ansteckungseffekter auch statistisch nachweisbar sind, hat eine USamerikanische Langfriststudie ergeben, die Netzwerkeffekte sehr präzise mit berücksichtigen konnte: »(Un)glückliche Nachbarn, Freunde und Verwandte wirkten auf die eigene Lebenszufriedenheit verstärkend zurück» (Fowler/Christiakis 2008).

\section{Fazit}

Wenn eine wieder zunehmende Arbeitslosigkeit ausschließlich unter dem Gesichtspunkt des Einkommensverlustes debattiert wird, bleiben wichtige Nebenwirkungen ausgeblendet. Die Regionalforschung zeigt, dass neben den erheblichen Wohlfahrtsverlusten für die Betroffenen selbst auch noch viele negative Zusatzwirkungen bestehen, die von einem allgemeinen Verlust an Zufriedenheit bis zu sinkenden Schulleistungen reichen. Unbekannt war 
bisher, ob auch die Lebenserwartung davon tangiert ist. Eine erste Analyse ergab, dass dies in Deutschland für beide Geschlechter nachweisbar ist, für Männer stärker als für Frauen. Der Verlust an Lebensjahren ist dabei beachtlich.

In einer so dramatischen Krise wie der gegenwärtig durchlebten, kann die politische Antwort darauf nie im Beharren auf nur einer Maßnahme liegen. Aber unter all den sicher wichtigen und aktuell angemessenen - nachfragestimulierenden Aktivitäten sollte nicht vergessen werden, dass auch in einer aktiven Arbeitszeitpolitik ein großes Potential zur Bekämpfung von Arbeitslosigkeit liegt. Denn hier kombiniert sich eine Arbeitsumverteilung zugunsten Erwerbsloser mit einer Hebung des Zufriedenheitsniveaus unfreiwillig überbeschäftigter Erwerbstätiger (Grözinger et al. 2008).

\section{Literatur}

Baltagi, B.H., Blien, U., Wolf, K. (2000): The East German wage curve 1993 - 1998, in: Economics Letters, 69(I), 25-3I.

Baltagi, B.H., Blien, U., Wolf, K. (2008): New Evidence on the Dynamic Wage Curve for Western Germany: 1980 - 2004, IZA Discussion Paper, No. 3433.

Baumert, J., Carstensen, C.H., Siegle, T. (2005): Wirtschaftliche, soziale und kulturelle Lebensverhältnisse und regionale Disparitäten des Kompetenzerwerbs, in: Deutschland, P.-K. (Hg.), PISA 2003. Der zweite Vergleich der Länder in Deutschland-Was wissen und können Jugendliche?, Münster, 323-366.

Blanchflower, D.G., Oswald, A.J. (1994): The Wage Curve, Cambridge MA: MIT Press.

Bundesamt für Bauwesen und Raumordnung (2004): INKAR, Indikatoren, Karten und Grafiken zur Raumentwicklung in Deutschland und Europa, Bonn.

Cromm, J., Scholz, R.D. (Hg.) (2002): Regionale Sterblichkeit in Deutschland, Göttingen.

De Vogli, R., Mistry, R., Gnesotto, R., Cornia, G.A. (2005): Has the relation between income inequality and life expectancy disappeared? Evidence from Italy and top industrialized countries, in: Journal of Epidemiology and Community Health, 59, I58 - I62.

Diez-Roux, A. (2002): A glossary for multilevel analysis, in: Journal of Epidemiology and Community Health, 56, $588-594$.

Ebenrett, H.J., Hansen, D., Puzicha, K.J. (2003): Verlust von Humankapital in Regionen mit hoher Arbeitslosigkeit, in: Aus Politik und Zeitgeschichte, B6/7, 25-3I.

Falk, A., Zweimüller, J. (2005): Unemployment and right-wing extremist crime, IZA Discussion Paper, No. I540.

Fowler, J.H., Christiakis, N.A. (2008): Dynamic spread of happiness in a large social network: Longitudinal analysis over 20 years in the Framingham Heart Study, in: British Medical Journal, 337(a2338), I - 9.

Grözinger, G. (2002): Makroökonomik der Seele? Über den Zusammenhang von Ungleichheit, Sozialkapital und Gesundheit, in: Ötsch, W., Panther, S. (Hg.), Ökonomik und Sozialwissenschaft. Ansichten eines in Bewegung geratenen Verhältnisses, Marburg: Metropolis Verlag, II9 - I53. 
Grözinger, G., Matiaske, W. (2004): Regional unemployment and individual satisfaction, in: Grözinger, G., van Aaken, A. (Hg.), Inequality: New Analytical Approaches, Marburg: Metropolis Verlag, 87 - IO4.

Grözinger, G., Matiaske, W., Tobsch, V. (2008): Arbeitszeitwünsche, Arbeitslosigkeit und Arbeitszeitpolitik, in: WSI Mitteilungen, 2, $92-99$.

Hewston, M., Antaki, C. (I990): Attributionstheorie und soziale Erklärungen, in: Stroebe, W., Hewstone, M., Codol, J.-P., Stephenson, G.M. (Hg.), Sozialpsychologie. Eine Einführung, Berlin, II2 - I43.

Kawachi, I., Kennedy, B.P., Glass, R. (1998): Social capital and self-rated health: A contextual analysis, in: American Journal of Public Health, 89(8), II87 - II94.

Kawachi, I., Kennedy, B.P., Wilkinson, R.G. (Hg.) (1999): The Society and Population Health Reader, New York: The New Press.

Kibele, E. (2008): Determinanten von regionalen Mortalitätsunterschieden in der Rentnerbevölkerung, in: Bund, D.R. (Hg.), Etablierung und Weiterentwicklung: Bericht vom vierten Workshop des Forschungsdatenzentrums der Rentenversicherung, Bad Homburg, I 43 - 156 .

Layard, P.R.G. (2005): Happiness: Lessons from a New Science, London: The Pengiun Press.

Legewie, J. (2008): Zum Einfluss regionaler Arbeitslosigkeit auf Einstellungen zur sozialen Gerechtigkeit, in: Kölner Zeitschrift für Soziologie und Sozialpsychologie, 6o(2), 286 - 313 .

Luechinger, S., Meier, S., Stutzer, A. (2008): Why does unemployment hurt the employed? Evidence from the life satisfaction gap between the public and the private sector, IZA Discussion Paper, No. 3385 .

Martin, A., Düll, H. (200o): Betriebliche Weiterbildung und Arbeitsmarktsituation: Qualifizierungsmaßnahmen im Lichte organisationstheoretischer Ansätze und empirischer Datenanalyse, in: Matiaske, W., Mellewigt, T., Stein, F.A. (Hg.), Empirische Organisations- und Entscheidungsforschung, Heidelberg, 8I - I23.

Nonnenmacher, A. (2007): Eignen sich Stadtteile für den Nachweis von Kontexteffekten? Eine empirische Analyse von Disorder und Kriminalitätsfurcht, in: Kölner Zeitschrift für Soziologie und Sozialpsychologie, 59(3), 493-5II.

Prognos (2008): engagementatlasog, Basel.

Ragnitz, J. (2006): Demographische Entwicklung in Ostdeutschland, Forschungsauftrag des BMWi, Endbericht, Halle.

Robert-Koch-Institut (2005): Armut, soziale Ungleichheit und Gesundheit: Expertise des Robert Koch-Instituts zum 2. Armuts-und Reichtumsbericht der Bundesregierung.

Robinson, W.S. (1950): Ecological correlations and the behaviour of individuals, in: American Sociological Review, I5(3), 35I - 357 .

Sanmartin, C., Ross, N.A., Tremblay, S., Wolfson, M., Dunn, J.R., Lynch, J. (2003): Labour market income inequality and mortality in North American metropolitan areas, in: Journal of Epidemiology and Community Health, 57(IO), $792-797$.

Schwarze, J., Andersen, H.H., Anger, S. (2002): Self-rated Health and Changes in Self-rated Health as Predictors of Mortality - First Evidence from German Panel Data, Humboldt- 
Universität zu Berlin, Wirtschaftswissenschaftliche Fakultät, Sonderforschungsbereich 373: Quantification and Simulation of Economic Processes.

Tarazona, M. (2007): Regionale Bildungsstrukturen und Beschäftigung in Deutschland, Discussion Papers, Universität Flensburg, Internationales Institut für Management, Nr. I6.

Wendt, C., Wolf, C. (Hg.) (2006): Soziologie der Gesundheit, in: Kölner Zeitschrift für Soziologie und Sozialpsychologie, Sonderheft 46, Köln.

Wilkinson, R.G. (1996): Kranke Gesellschaften. Soziales Gleichgewicht und Gesundheit, Wien und New York.

Wilkinson, R.G. (2005): The Impact of Inequality. How to Make Sick Societies Healthier, New York: The New Press.

Wilkinson, R.G., Pickett, K.E. (2006): Income inequality and population health: A review and explanation of the evidence, in: Social Science \& Medicine, 62, 1768 - I784.

Wilkinson, R.G., Pickett, K.E. (2007): The problems of relative deprivation: Why some societies do better than others, in: Social Science \& Medicine, 65, 1965 - 1978.

Windzio, M. (2004): Kann der regionale Kontext zur >Arbeitslosenfalle Zeitschrift für Soziologie und Sozialpsychologie, 56(2), 257- 278.

\section{Divergences in EMU: Scope of the problem and policy options Sebastian Dullien*}

\section{Introduction}

Ten years after the introduction of the euro, the question of economic divergence has gained new prominence. While immediately after the introduction of the euro, skeptical voices about the young currency's sustainability fell silent, the debate first reemerged in the years after the bursting of the New-Economy-bubble in 2000. With Italy and Germany underperforming the rest of the euro-area in the recovery after 200I, think-tanks and investment banks debated whether economic divergence might in the end lead to a break-up of the currency union (Gros 2006, Munchau 2006, Prior-Wandesforde/Hacche 2005).

After shortly submerging in the years of robust economic growth in 2007 and 2008 , the debate reemerged once again in early 2009 with rating agencies downgrading or putting on watch Ireland's, Portugal's and Spain's sovereign debt and interest rate spreads between euro-area countries widening to levels not observed since the start of EMU. Now, it is Spain, Ireland and Greece (which had outperformed the euro-area in prior years) which are seen as those at risk of leaving the currency union or being forced out of it by financial markets.

* HTW Berlin.

C INTERVENTION 6 (I), 2009, 24-32 